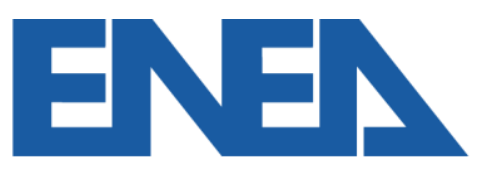

Agenzia nazionale per le nuove tecnologie,

l'energia e lo sviluppo economico sostenibile

\title{
Wavefront control in the design of narrow- and broad-band optical coatings
}

Angela Piegari, Anna Sytchkova, ENEA, Roma, Italy

Matteo Taccola, ESA-ESTEC, Noordwijk, The Netherlands

ICSO2018-12/10/2018 


\section{Outline}

- Optical Coatings and Wavefront deformation

- Historical notes

- Wavefront profile and spectral behavior

- Narrow-band and broad-band coatings

- Wavefront optimization criteria

- Simple examples of mirrors and filters

- Conclusions 


\section{Scope of the work}

The wavefront deformation can be caused by several factors, for example stress and consequent bending of the optical element or refractive index inhomogeneities, which require dedicated solutions.

The aspect considered here is the effect caused by the coating thickness non-uniformity, which depends on the manufacturing process. This effect has often been underestimated with serious consequences in the performance of optical instruments.

The aim of this work is:

$\checkmark$ to analyze the wavefront distortion caused by undesired spatial thickness variations, in both narrow-band and broad-band coatings

$\checkmark$ to study the wavefront spectral behaviour in the wavelength range of interest

$\checkmark$ to identify simple solutions for reducing the wavefront distortion in mirrors and filters 


\section{Background}

from P. Kupinski, A. Macleod : Advances in optical manufacturing, Laser Focus World, 2015

"In extremely high-quality imaging and interferometric systems, the change in phase - especially from a reflecting coating - can become a performance issue, as the wavefront at any given wavelength is a function of both surface figure and the phase change induced by the coating "

"The reflected phase from an interference coating can change quickly with, otherwise spectrally insignificant changes in coating thickness or index of refraction "

a) $0.26 \lambda \mathrm{P}-\mathrm{V}$

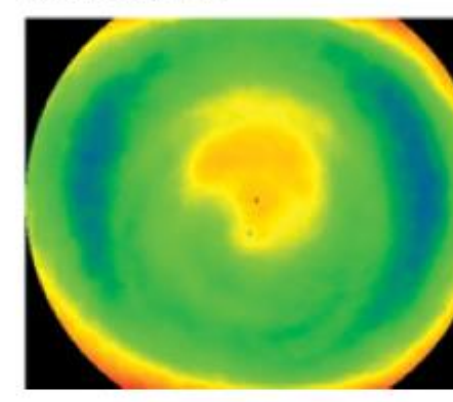

Form (peak-to-valley) of a fused silica mirror: pre-coat (a), after applying a multilayer broadband dielectric mirror (b),

Early studies of the effect of coating non-uniformity on the phase of reflected beams P. Giacomo, Propriétés chromatiques des couches réfléchissantes multi-diélectriques, J. Phys. Radium 1958

Proposed design of wideband dielectric mirror with phase compensation

P. Ciddor, Minimization of the Apparent Curvature of Multilayer Reflecting Surfaces, Appl. Opt. 1968 


\section{Wavefront: non uniform coatings}

Wavefront: locus of all points having the same phase along the direction of wave propagation.

Phase difference along the coating radius:

$\Delta p h^{R}(r)=\varphi^{R}(r)-\varphi^{R}(0)+4 \pi[t(r)-t(0)] / \lambda$ $\Delta p h^{T}(r)=\varphi^{T}(r)-\varphi^{T}(0)+2 \pi[t(r)-t(0)] / \lambda$

where $\varphi^{R, T}$ is the reflected or transmitted coating phase, $r$ is the radius, $\lambda$ is the wavelength, $t$ is the coating thickness.

reference plane

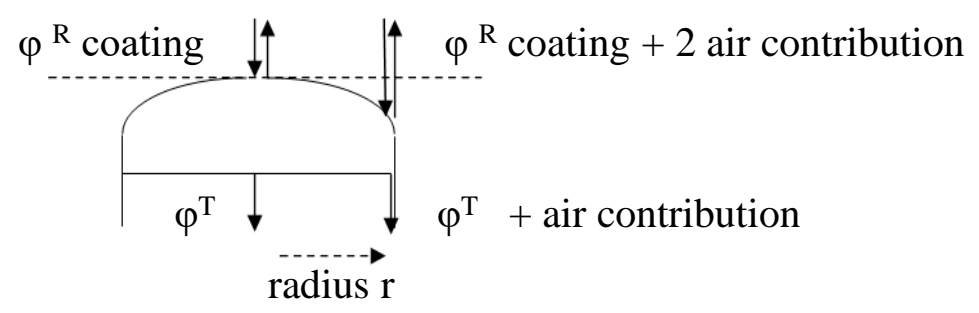

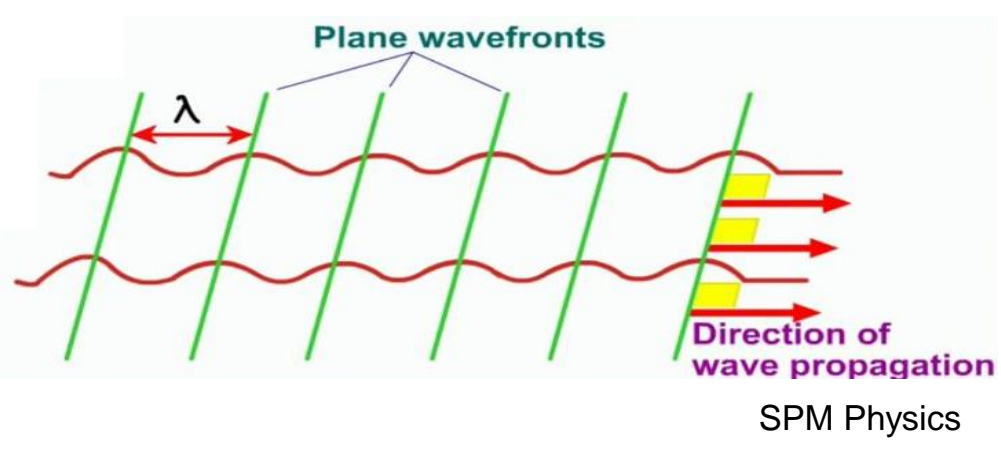

TRANSMITTED WAVEFRONT DISTORTION
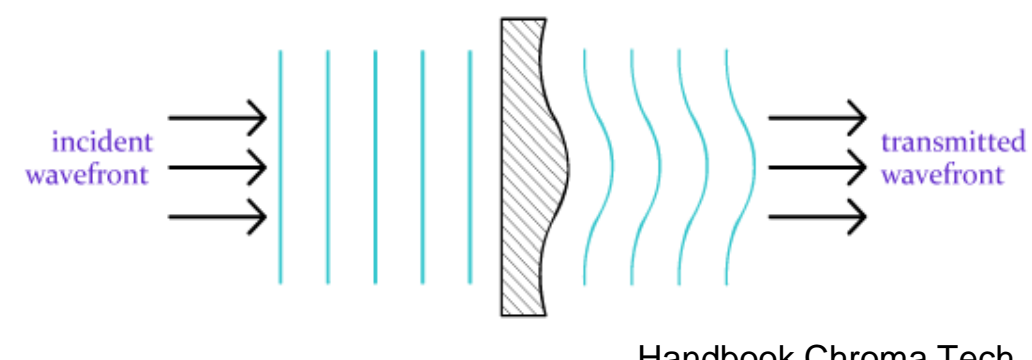

Handbook Chroma Tech

$\Delta p h$ should be zero to have a plane wavefront 


\section{Wavefront: narrow and broadband coatings}

The geometrical wavefront is determined by the decrease of thickness (path of the beam in air), the actual wavefront includes the contribution of interference

The wavefront in reflection undergoes a greater distortion than in transmission (the path of the beam in air is doubled).

The examples reported in the following are mainly dedicated to reflecting coatings

Two categories are analized:

$>$ narrow-band dielectric mirrors

$>$ broad-band coatings (behavior in a wide wavelength range) 


\section{Wavefront: quater-wave mirrors}

\section{A typical high-reflectance mirror is made of quarter-wave layers}

Example: $\lambda_{0}=600 \mathrm{~nm}, \mathrm{SiO}_{2}$ and $\mathrm{Ta}_{2} \mathrm{O}_{5}$,

$2 \%$ of thickness radial reduction with parabolic profile

19 layers: Sub/HLHL...H/Air (R=99.9\%)

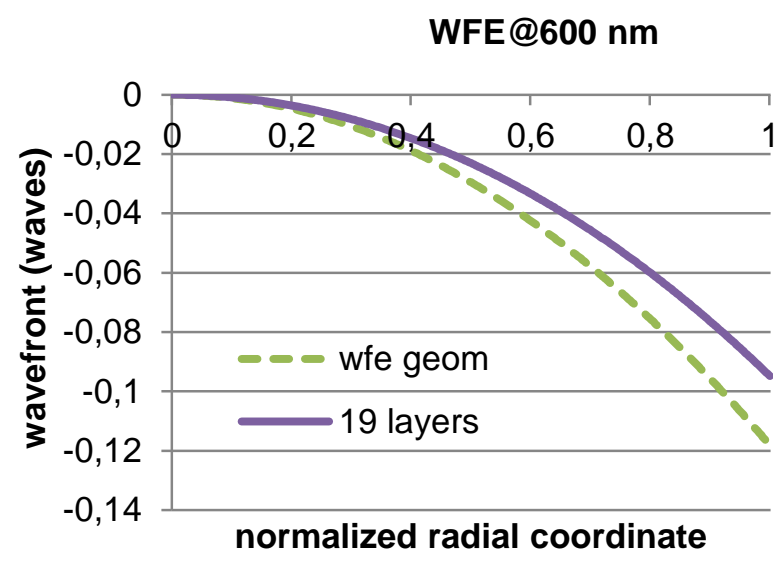

18 layers: Sub/HLHL...L/Air (R=99.5\%)

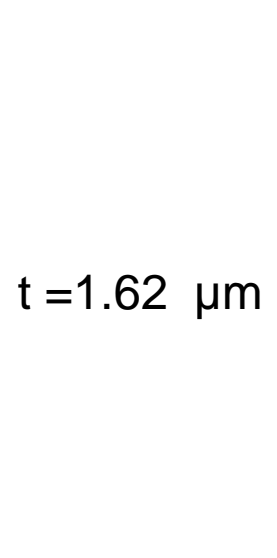

WFE @600 nm

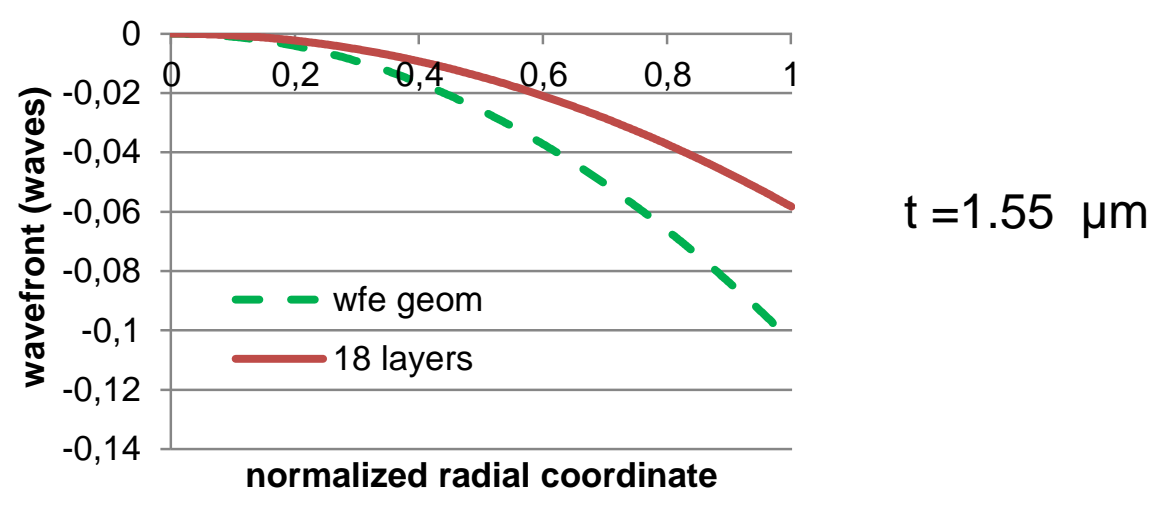

It appears that the structure ending with a layer $L$ (at the air side) is more favorable (WFE changes from 0.1 waves to 0.06 waves $P V$ ). 


\section{Coating thickness and reflected phase}

It has been demonstrated that an almost perfect compensation of the phase difference can be reached if the following relation is satisfied:

$$
\partial \varphi / \partial \sigma=-4 \pi t
$$

where $\sigma$ is the wavenumber $(1 / \lambda)$ and t the total coating thickness (P.Giacomo).

The slope of phase in the reflection band, near the central wavelength $\lambda_{0}$, is given by the following formula, valid for periodic structures. ${ }^{*}$

$$
\begin{array}{ll}
\text { last layer } \mathrm{H} & \frac{\partial \varphi}{\partial \sigma}=\frac{-\pi \lambda 0}{n H-n L} \\
\text { last layer } \mathrm{L} & \frac{\partial \varphi}{\partial \sigma}=\frac{-\pi \lambda 0 n H n L}{n H-n L}
\end{array}
$$

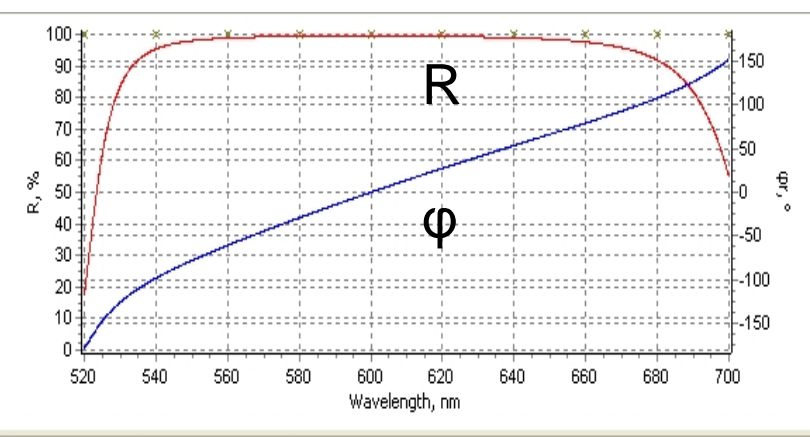

It is possible to act on the number of layers (total thickness) and the refractive indices to reach an almost plane wavefront in a dielectric non-uniform mirror.

Typically high values of $\mathrm{n}_{\mathrm{H}}$ and $\mathrm{n}_{\mathrm{L}}$ are needed to obtain a plane wavefront together with a high reflectance.

* Baumeister, P.W., [Optical coating technology], SPIE press, Bellingham WA, sections 5 and 10 (2004) 


\title{
Wavefront spectral behavior: narrow-band mirror
}

\author{
q.w. mirror 22 layers, $\mathrm{n}_{\mathrm{H}}=2.35\left(\mathrm{TiO}_{2}\right), \mathrm{n}_{\mathrm{L}}=1.9\left(\mathrm{Y}_{2} \mathrm{O}_{3}\right)$, thickness $1.57 \mu \mathrm{m}$
}

WFE radial behavior at a single wavelength

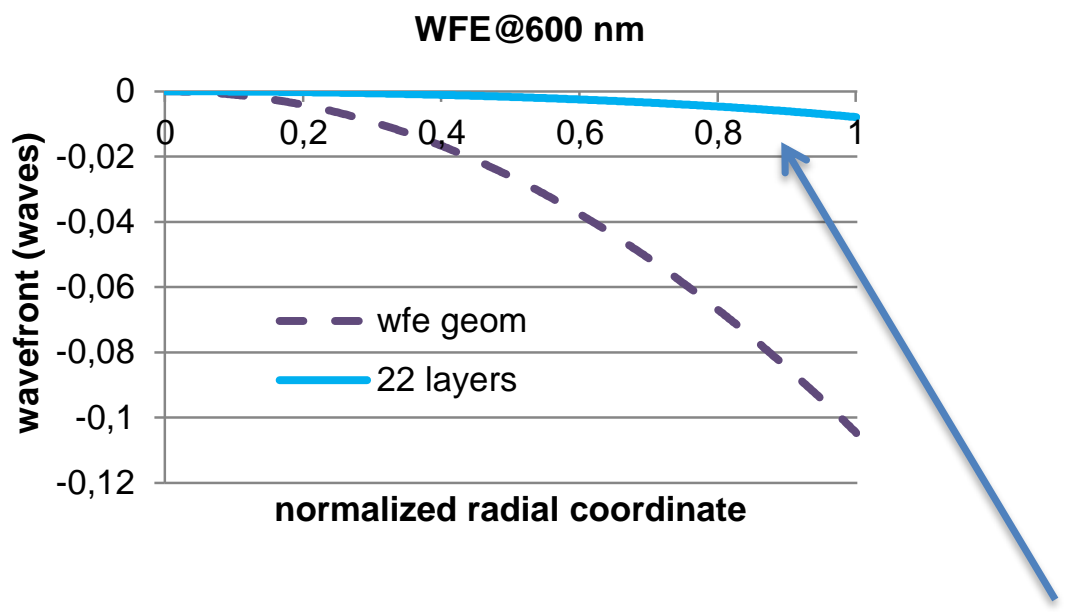

WFE spectral behavior with $2 \%$ thickness decrease

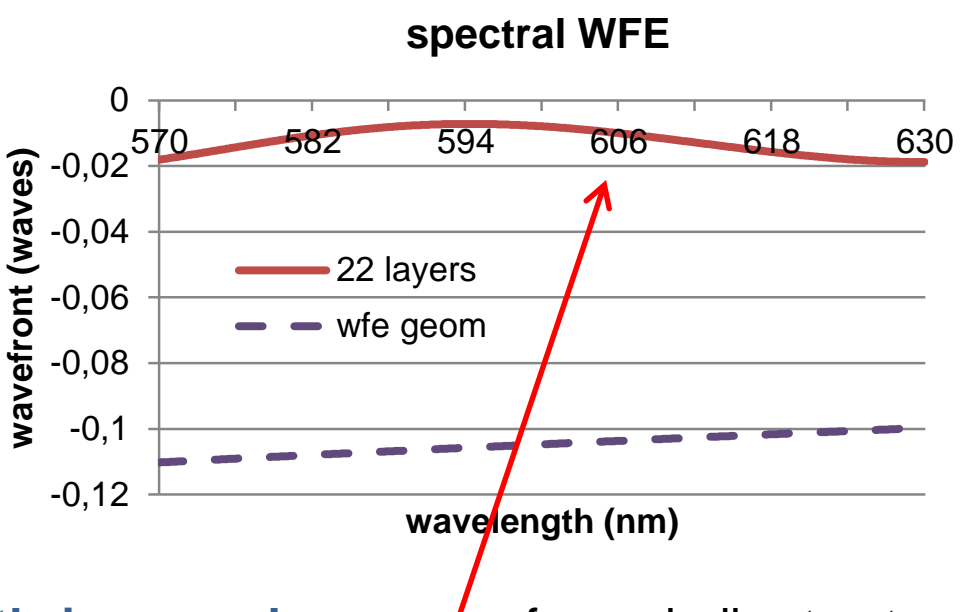

The wavefront at the central wavelength is significantly improved, moreoyer for periodic structures, as the quarter-wave coatings, the wavefront spectral profile is almost constant in the reflectance band 


\section{Broad-band mirror: phase behavior}

\section{Broad-band mirror:}

combination in the same stack of two or more coatings in series centered at

different wavelengths (or a gradual change of thicknesses through the structure)

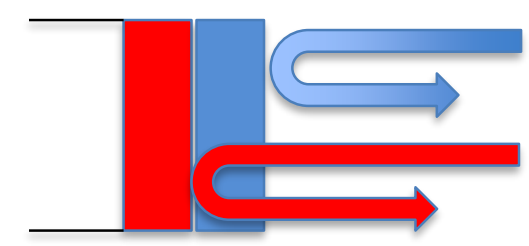

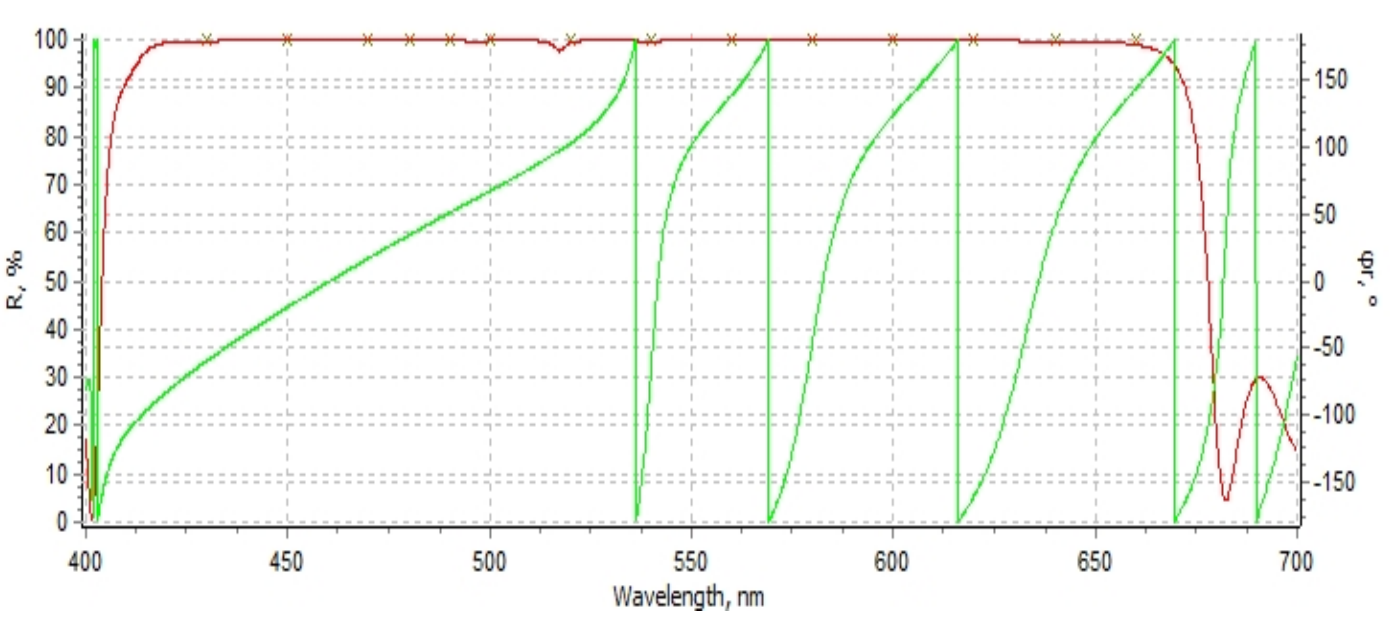

46 layers $\left(\mathrm{SiO}_{2}, \mathrm{Ta}_{2} \mathrm{O}_{5}\right), \mathrm{t}=3.5 \mu \mathrm{m}, \mathrm{R}>99 \%$ in VIS range: reflected intensity (red), reflected phase (green)
The stack with the lower central wavelength is typically the external one on the air side to avoid absorption, thus is the first structure encountered by the beam that will be totally reflected at this short wavelengths.

The long wavelengths penetrate into the coating before being returned and that causes a significant phase effect (the situation is reversed if the stack centered at longer wavelengths is positioned on the outer side). 


\section{Radial and spectral behavior in a broad-band mirror}

\section{6-layer mirror: wavefront change with wavelength (chromatic aberration)}

spectral wfe

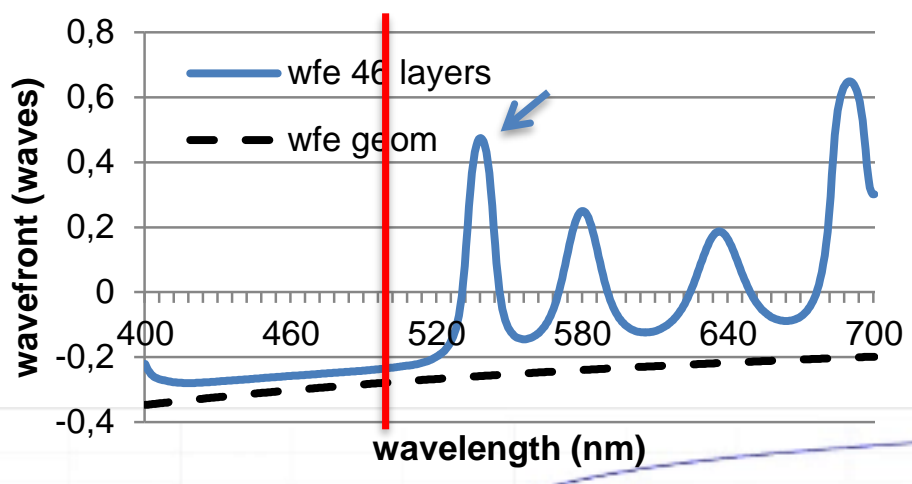

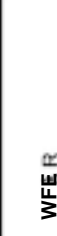

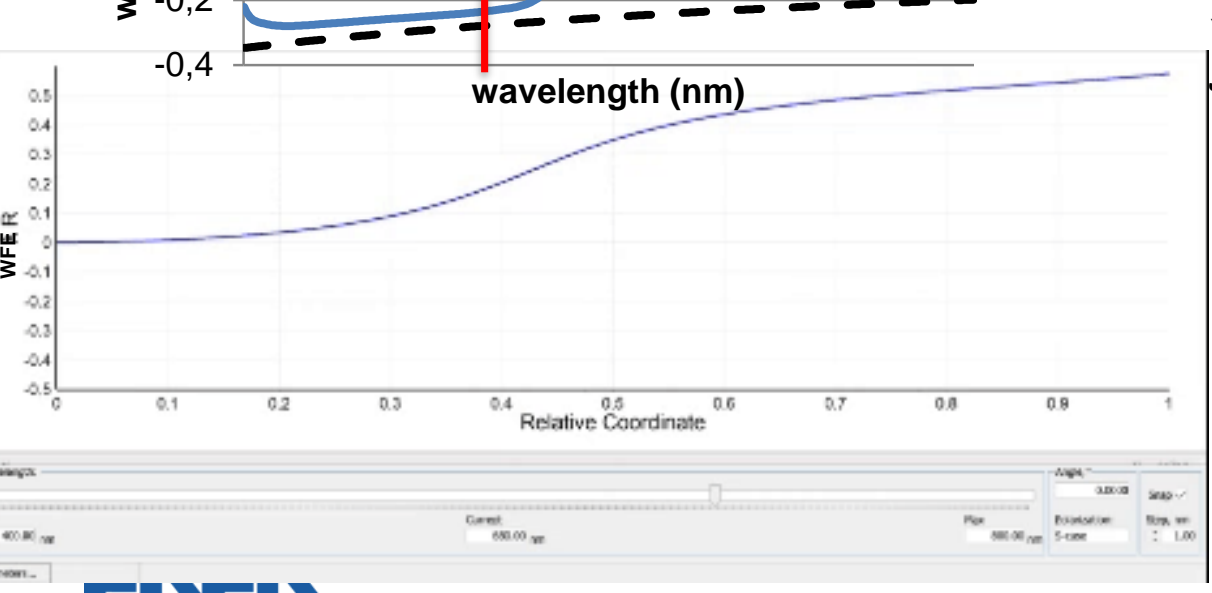

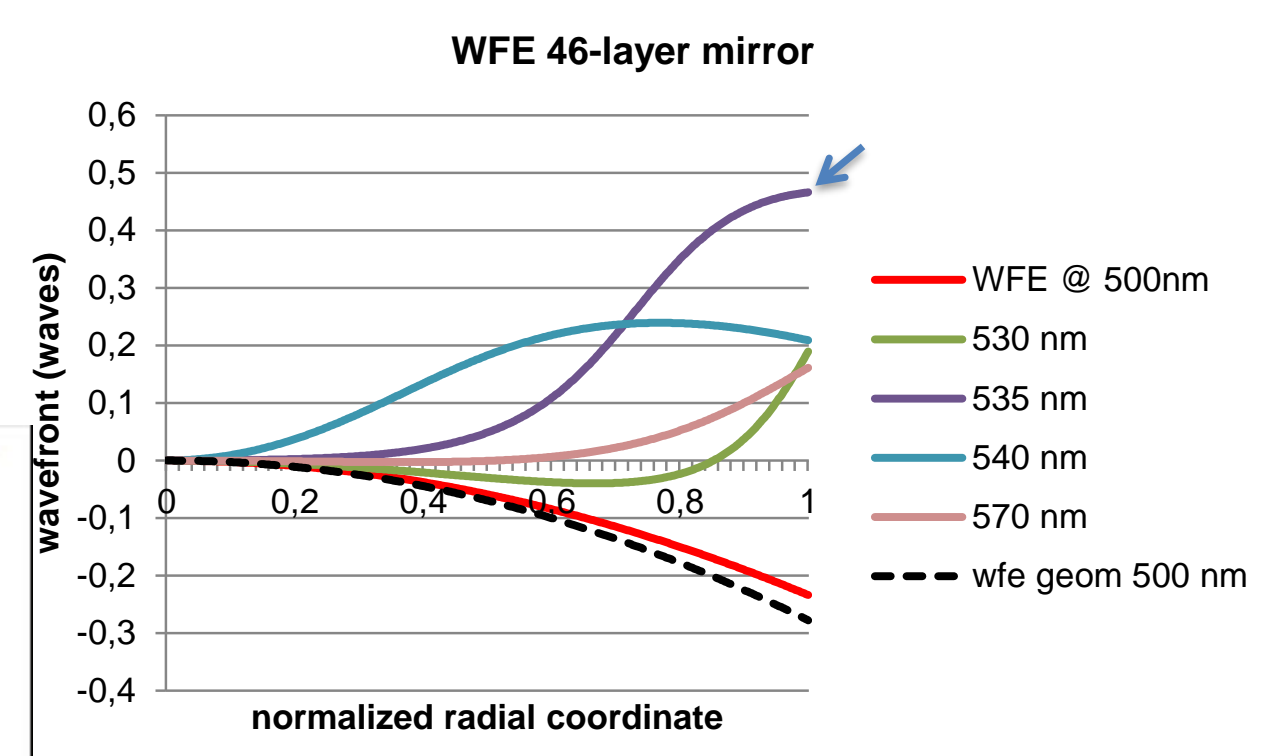

max WFE 0.5 waves at $\lambda=535 \mathrm{~nm}$ 


\section{Wavefront deformation in a telescope}

\section{Wavefront deformation induced in the instrument exit beam}

An example of the effect that a broad-band mirror can induce in a telescope (TMA with intermediate focal plane, largely used in space instrumentation) is analyzed using the 46-layer mirror for the small folding mirror before the focal plane.

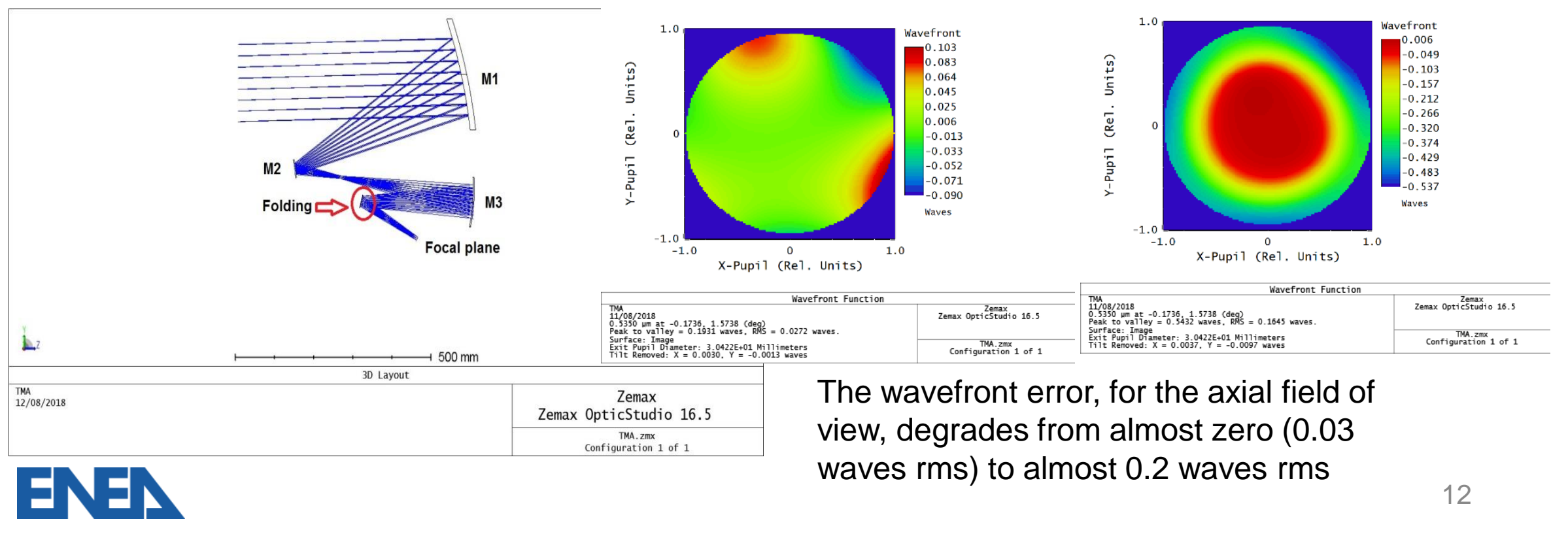




\section{Wideband mirror: modified design}

46-layer q.w mirror $\left(\mathrm{SiO}_{2}, \mathrm{Ta}_{2} \mathrm{O} 5\right)$ only two layer thickness modified (the central and the outer layer)
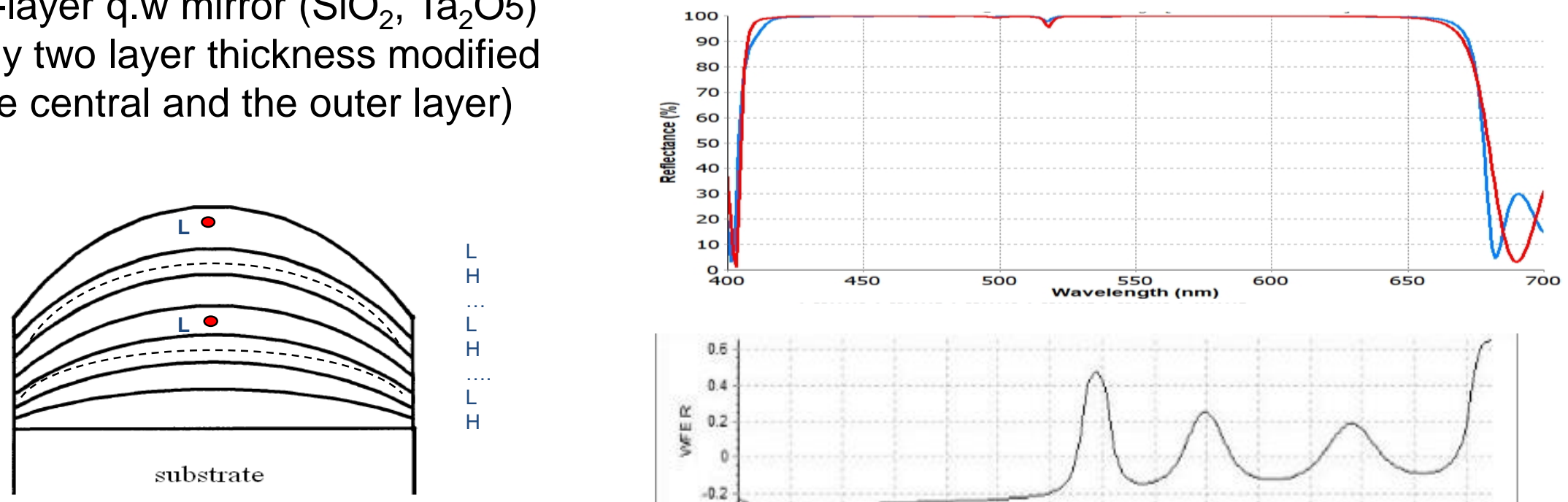

max WFE (2\% thickness decrease) changes from 0.5 to 0.3 waves
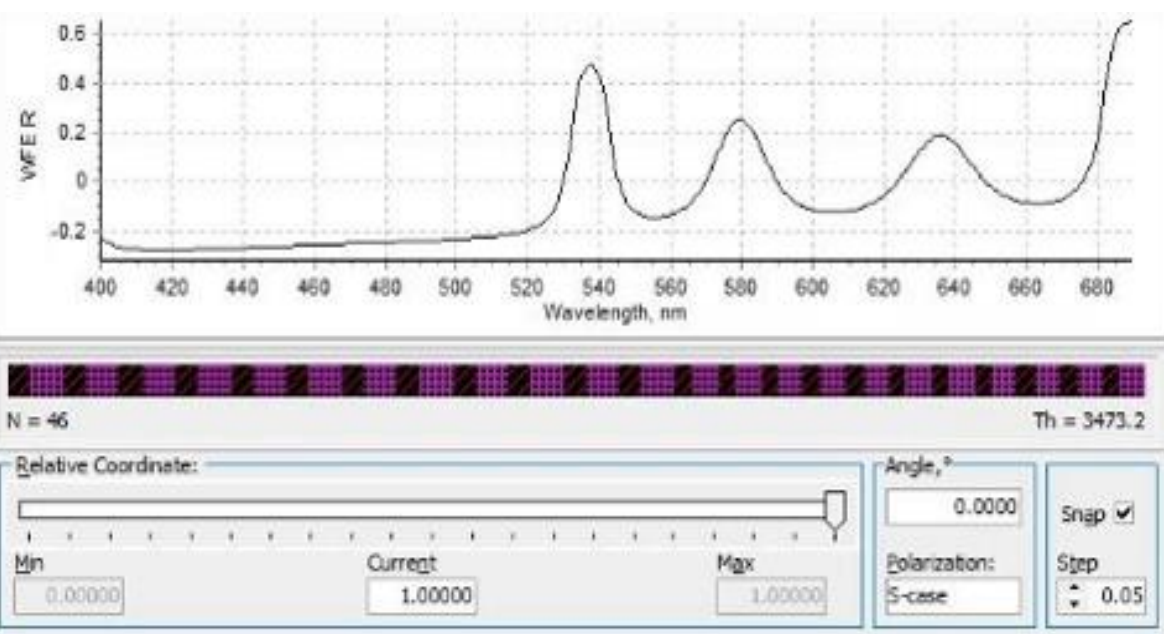


\section{Software methods}

$\square$ Coatings with target performance may be designed taking advantage of dedicated software.

$\square$ Coatings are typically optimized for the intensity of the reflected/transmitted beams. Optimization of the phase behavior is rarely required; for example phase derivative with respect to the frequency (group delay dispersion, GDD) is essential in chirped mirror design.

a A good analytical way of designing coatings with unperturbed wavefront is not available, but refinement and synthesis methods may provide reasonable results.

In the case of non uniform coatings, which induce wavefront deformation, the wavefront control could be made by combining in the merit function several parameters:

- High reflectance for wideband mirrors (or other intensity characteristics)

Flat wavefront profile over the wavelength range of interest

- Value of the WFE equal zero 


\section{Wideband mirror: optimized design}

- 46-layer q.w. mirror and 42-layer optimized mirror (SiO2, Ta2O5), t 3.5 $\mu \mathrm{m}$

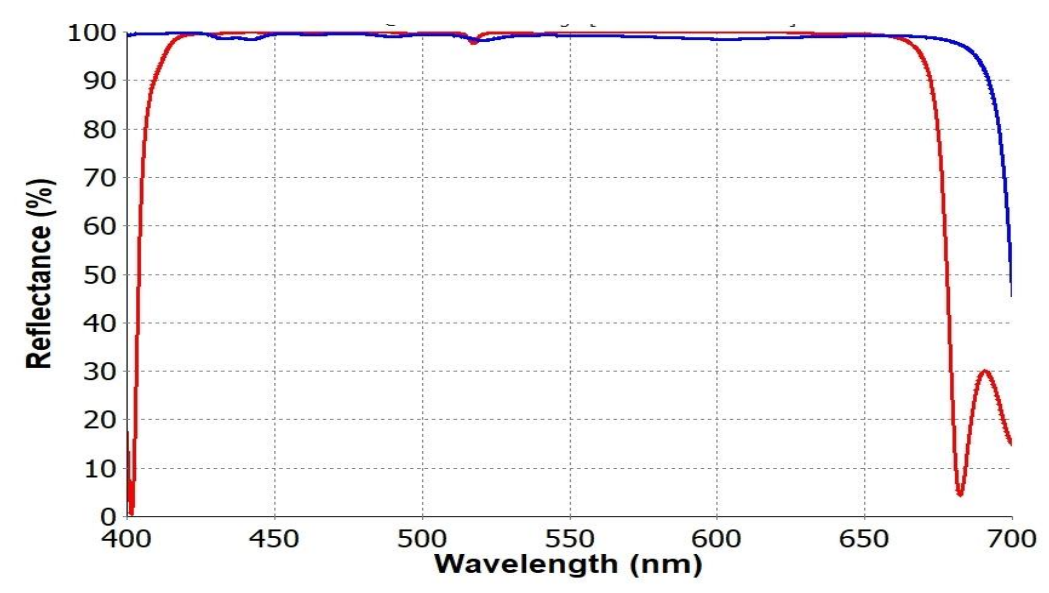

Spectral WFE with 1\% thickness decrease

Reflectance of the two mirrors: 46 layers (red), 42 layers (blue)

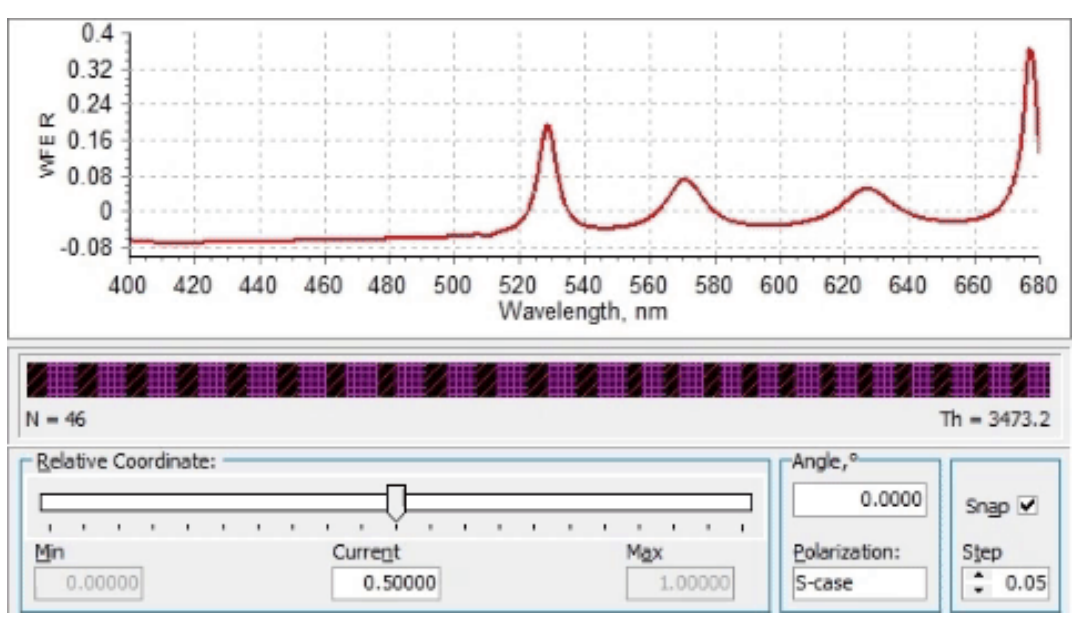

WFE max: 46 layers, 0.2 waves

42 layers, 0.08 waves without oscillations 


\section{Wideband mirror: optimized design}

- Reducing the number of layers and total thickness, the wavefront improves but a lower reflectance should be accepted

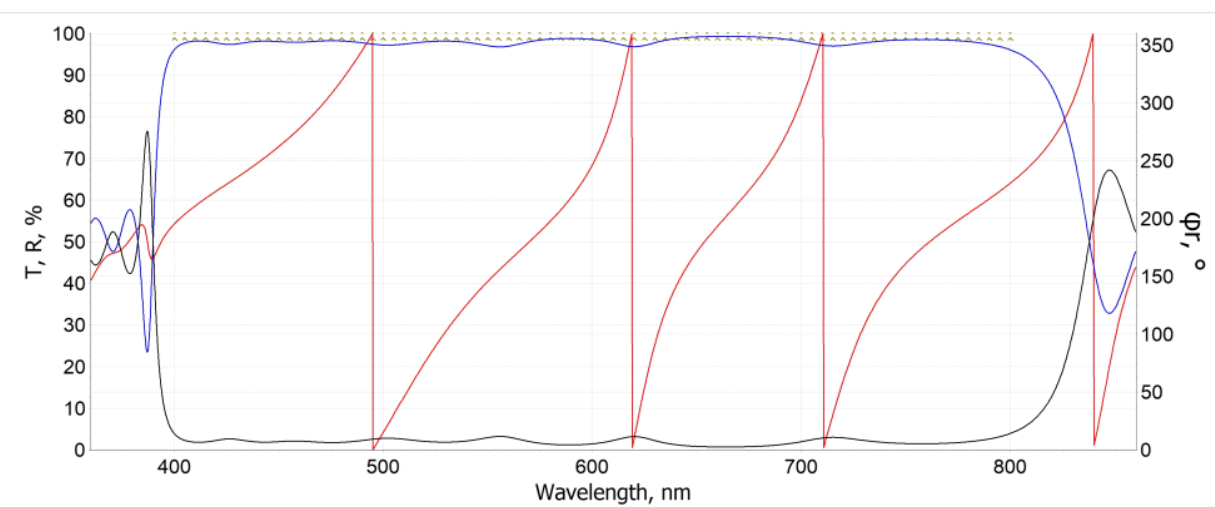

Reflectance, transmittance and phase of a 23-layer wideband mirror $(t=2 \mu \mathrm{m})$
Spectral WFE with $1 \%$ thickness decrease

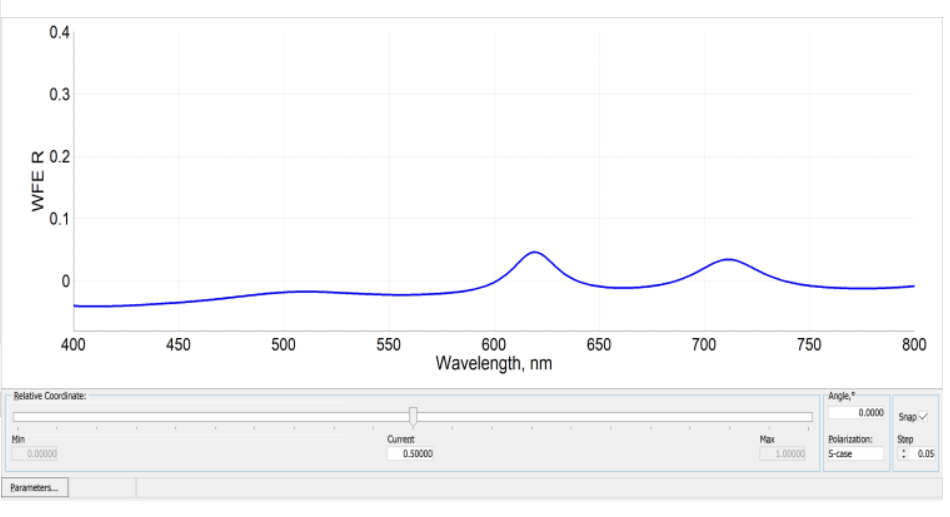

WFE max: 0.05 waves 


\section{Wavefront: metal mirrors}

Alternative solution: enhanced silver mirror

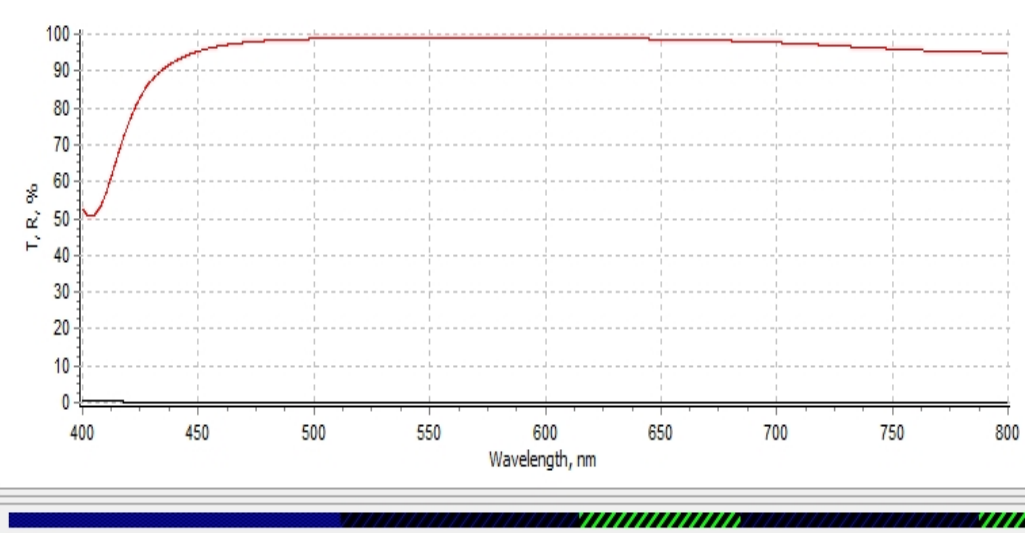

Spectral reflectance $\mathrm{R}>99 \%$ in the VIS range
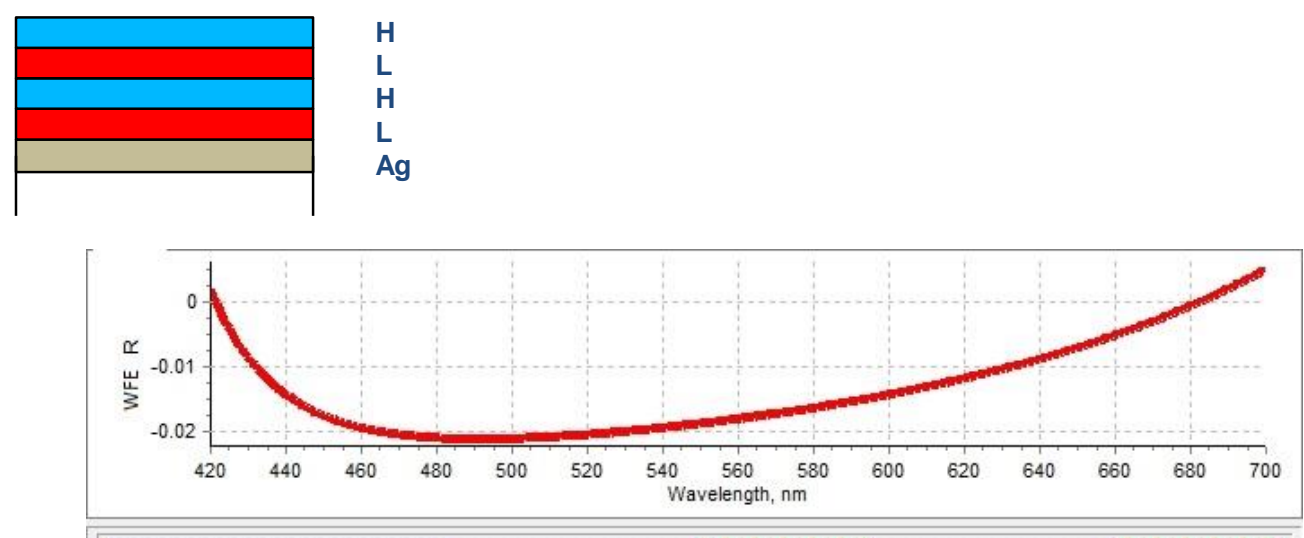

$\mathrm{N}=5$

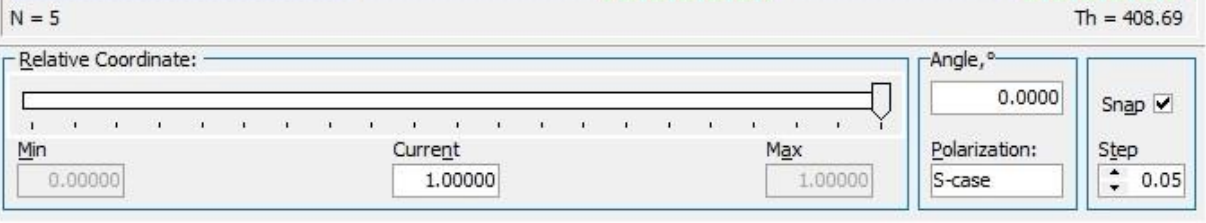

WFE versus wavelength, with $2 \%$ thickness variation

The wavefront deformation in this case is low (max WFE 0.02 waves). The limitation in its use could be given by the space environmental conditions 


\section{Transmitted wavefront}

The problem of the wavefront distortion in transmittance seems less critical. In a broad-band transmitter the light tends to go straight through and so the phase behaves as expected from a single pass and does not vary as much as it can in a reflector.

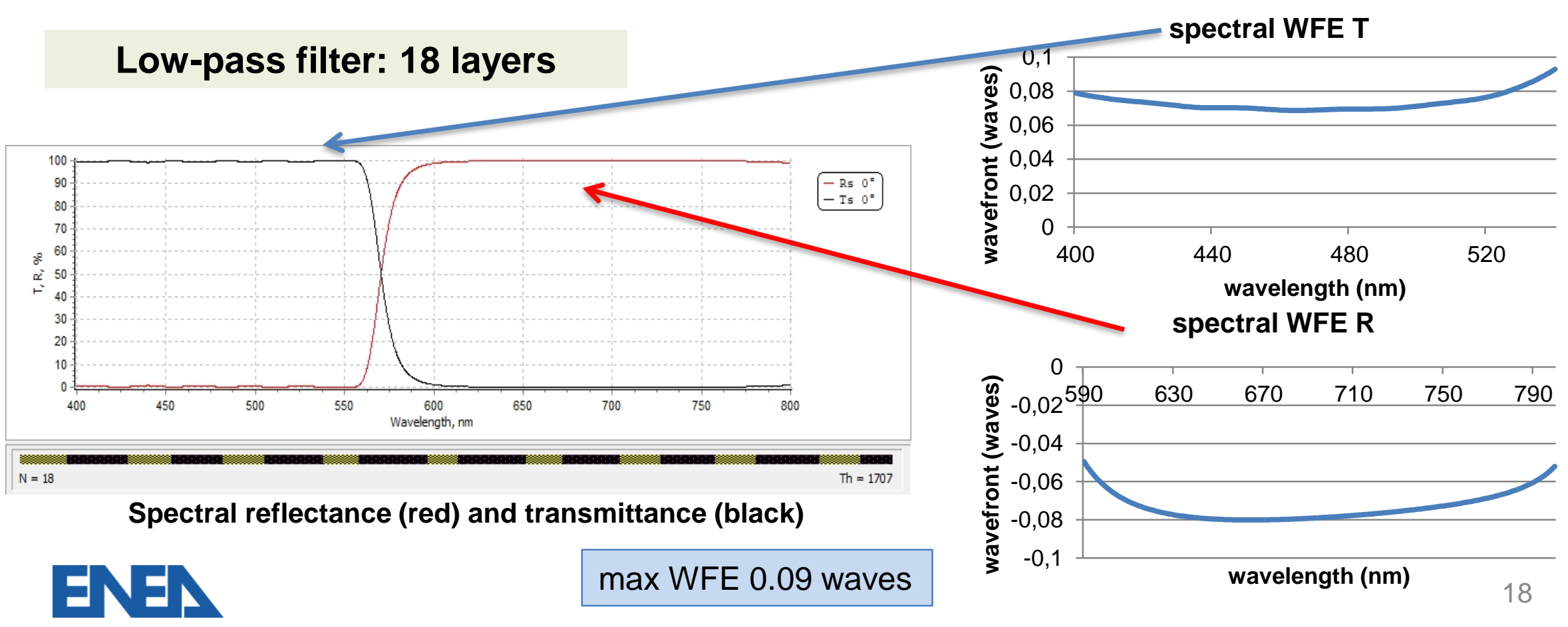




\section{Wavefront: Fabry-Perot filter}

LI: Double-cavity Fabry-Perot filter $\left(\mathrm{SiO}_{2}, \mathrm{TiO}_{2}\right)$

Central wavelength $777.8 \mathrm{~nm}$

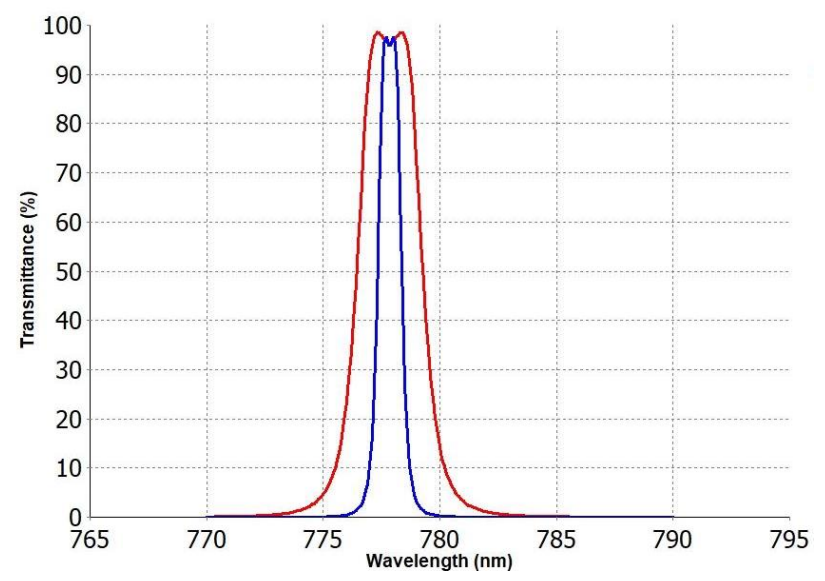

Spectral transmittance

Red: 35 layers $F W H M=3 \mathrm{~nm}, t=3.9 \mu \mathrm{m}$

Blue: 43 layers $F W H M=1 \mathrm{~nm}, t=4.7 \mu \mathrm{m}$
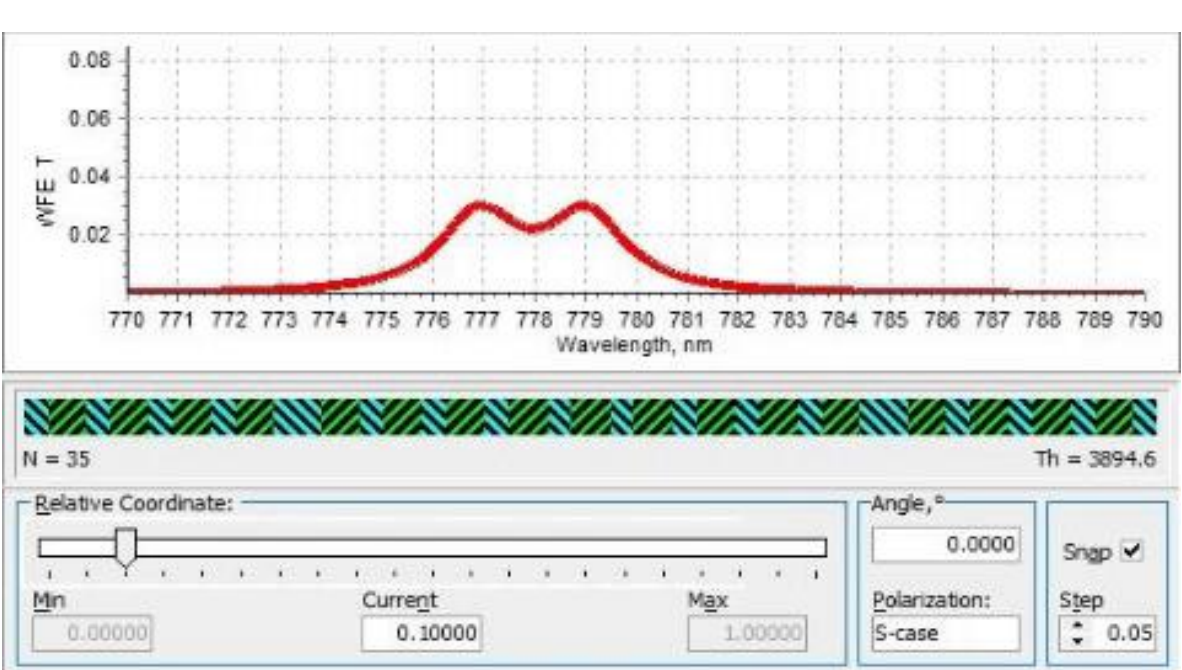

Spectral wavefront with a thickness non uniformity of $0.2 \%$ 


\section{Conclusions}

- The wavefront distortion caused by coating non uniformity has been known for many years, nevertheless the consequences are often underestimated.

Attention should be paid to this effect, especially in the case of highreflecting broadband mirrors.

- The wavefront improvement can be obtained by software with a proper coating design, however simple rules could be applied to reduce the effect of coating non-uniformity.

The impact of this problem on the instrument performance is dependent on the instrument configuration, and in some cases could be detrimental. 